\title{
Erratum: First Measurement of Charm Production in Its Fixed-Target Configuration at the LHC [Phys. Rev. Lett. 122, 132002 (2019)]
}

\author{
R. Aaij et al.
}

(LHCb Collaboration)

(Received 17 October 2019; published 2 December 2019)

\begin{abstract}
DOI: 10.1103/PhysRevLett.123.239901
\end{abstract}
In the original Letter, a mistake was made in the calculation of the $c \bar{c}$ production cross section $\sigma_{c \bar{c}}=288 \pm 24 \pm 7 \mu \mathrm{b} /$ nucleon in $p \mathrm{He}$ collisions at $\sqrt{s_{N N}}=86.6 \mathrm{GeV}$. The $D^{0}$ cross section $\sigma_{D^{0}}=156.0 \pm$ $13.1 \mu \mathrm{b} /$ nucleon has been measured by adding $K^{-} \pi^{+}$and $K^{+} \pi^{-}$final states to account for both $D^{0}$ and $\bar{D}^{0}$ decays. The $c \bar{c}$ cross section has then been obtained by dividing the measured $D^{0}$ cross section by the global fragmentation factor $f\left(c \rightarrow D^{0}\right)=0.542 \pm 0.024$, leading to $\sigma_{c \bar{c}}=288 \pm 24 \pm 7 \mu \mathrm{b} /$ nucleon, while it should have been divided by $2 \times f\left(c \rightarrow D^{0}\right)$, with the factor 2 accounting for the inclusion of both the $K^{-} \pi^{+}$and $K^{+} \pi^{-}$final states. Therefore, the quoted $c \bar{c}$ production cross section should be $\sigma_{c \bar{c}}=144 \pm 12 \pm 4 \mu \mathrm{b} /$ nucleon instead of $\sigma_{c \bar{c}}=288 \pm 24 \pm 7 \mu \mathrm{b} /$ nucleon, as stated in the original Letter. We include here Fig. 2, which replaces Fig. 2 of the original Letter (only the right plot has been changed).

In addition, the $D^{0}$ differential cross sections per target nucleon, as functions of $y^{*}$ and $p_{T}$ are also obtained by adding $K^{-} \pi^{+}$and $K^{+} \pi^{-}$final states. The HELAC-ONIA predictions, providing the $c \bar{c}$ cross section as functions of $y^{*}$ and $p_{T}$, have been scaled with $f\left(c \rightarrow D^{0}\right)$ in order to compare with our data. Here again, a factor 2 is missing. HELAC-ONIA predictions should have been scaled by $2 \times f\left(c \rightarrow D^{0}\right)$ in order to take into account the inclusion of both $K^{-} \pi^{+}$and $K^{+} \pi^{-}$final states in the determination of the $D^{0}$ cross section. Therefore, the HELAC-ONIA predictions, after proper correction, overestimate (instead of underestimate, as stated in the original Letter) the measured total cross section. The HELAC-ONIA predictions must be rescaled by a factor of 0.72 (instead of 1.44, as stated in the original Letter) to compare the shape of the distributions (experimental results stay unchanged).

We include here Fig. 4, which replaces Fig. 4 of the original Letter (only the top plots have been changed).
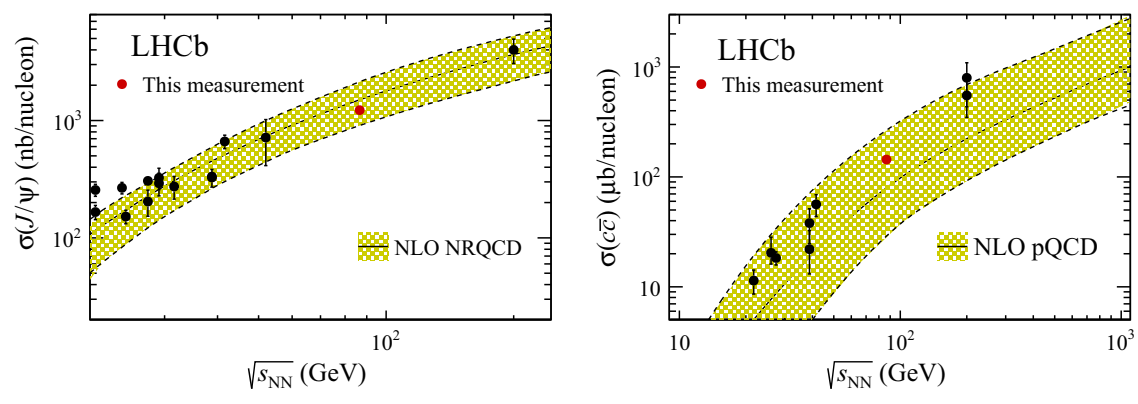

FIG. 2. (Left panel) $J / \psi$ cross-section measurements as a function of the center-of-mass energy. Experimental data, represented by black points, are taken from Ref. [1]. The band corresponds to a fit based on next-to-leading-order (NLO) nonrelativistic QCD (NRQCD) calculations [1]. (Right panel) $c \bar{c}$ cross-section measurements as a function of the center-of-mass energy. Experimental data, represented by black points, are taken from Ref. [2]. The yellow band corresponds to NLO perturbative QCD (pQCD) calculations [3]. The red points correspond to the $p \mathrm{He}$ results from the present analysis.

"Full author list given at the end of the original Letter.

Published by the American Physical Society under the terms of the Creative Commons Attribution 4.0 International license. Further distribution of this work must maintain attribution to the author(s) and the published articles title, journal citation, and DOI. 
PHYSICAL REVIEW LETTERS 123, 239901(E) (2019)
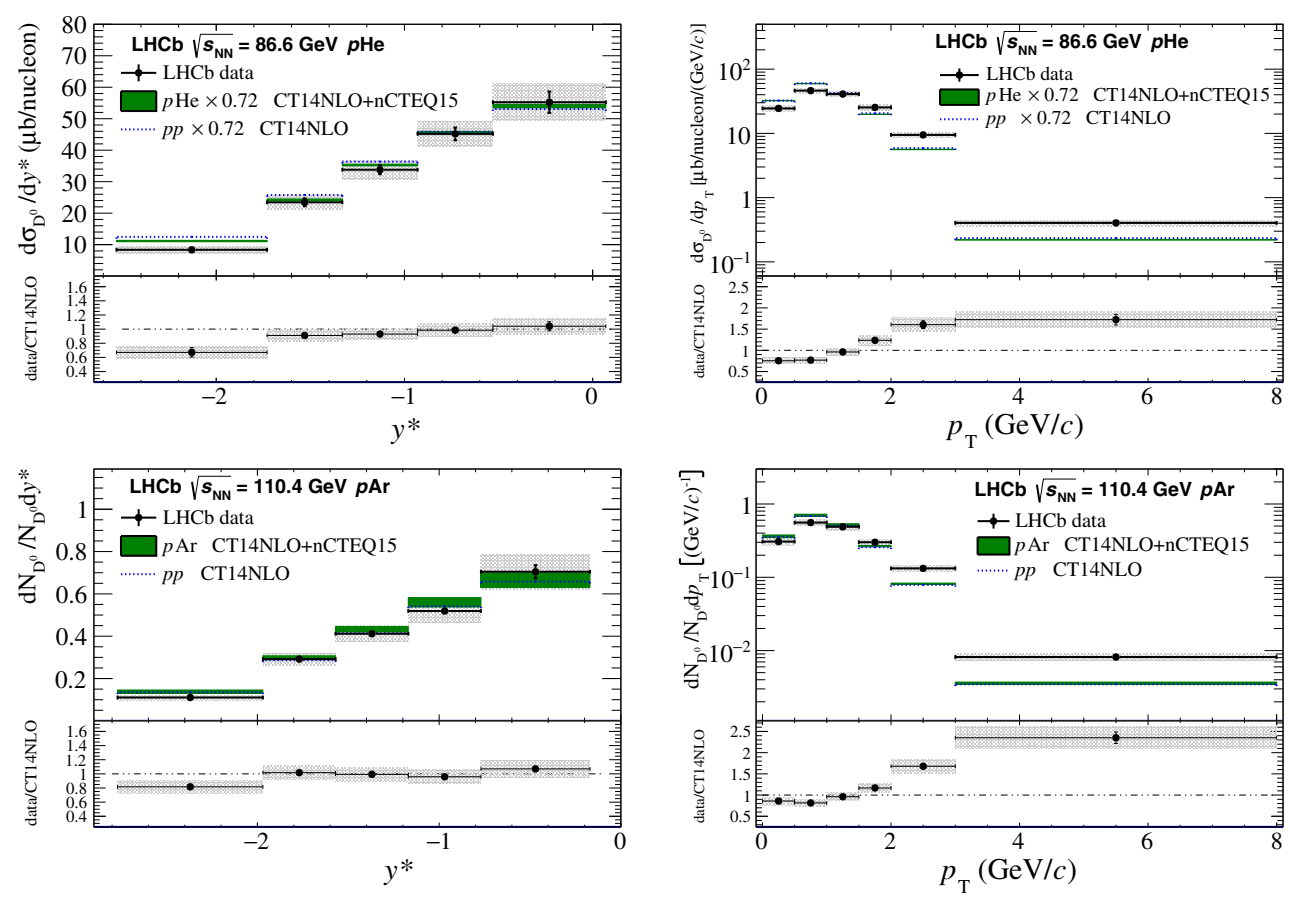

FIG. 4. Differential $D^{0}$ production cross sections for (top panels) $p$ He and differential $D^{0}$ yields for (bottom panels) $p$ Ar collisions, as a function of (left panels) center-of-mass rapidity $y^{*}$ and (right panels) transverse momentum $p_{T}$. The data points mark the bin centers. The quadratic sum of statistical and uncorrelated systematic uncertainties are indicated by the vertical black lines. The correlated systematic uncertainties are indicated by the gray area. Theoretical predictions are described in the text. The lower panel of each plot shows the ratio of the data to the HELAC-ONIA $p p$ predictions.

[1] F. Maltoni et al., Analysis of charmonium production at fixed-target experiments in the NRQCD approach, Phys. Lett. B 638, 202 (2006).

[2] J. Adam et al. (ALICE Collaboration), $D$-meson production in $p$ - $\mathrm{Pb}$ collisions at $\sqrt{s_{N \mathrm{~N}}}=5.02 \mathrm{TeV}$ and in $p p$ collisions at $\sqrt{s}=7$ TeV, Phys. Rev. C 94, 054908 (2016).

[3] M. L. Mangano, P. Nason, and G. Ridolfi, Heavy quark correlations in hadron collisions at next-to-leading order, Nucl. Phys. B373, 295 (1992). 